\title{
Vibroimpact Dynamics of a Tethered Satellite System
}

\author{
B. S. Yu and H. Wen \\ State Key Laboratory of Mechanics and Control of Mechanical Structures, \\ Nanjing University of Aeronautics and Astronautics, Nanjing 210016, China \\ Correspondence should be addressed to B.S. Yu; yu_bensong@nuaa.edu.cn
}

Received 26 April 2017; Revised 10 September 2017; Accepted 4 October 2017; Published 21 November 2017

Academic Editor: Carlo Trigona

Copyright (C) 2017 B. S. Yu and H. Wen. This is an open access article distributed under the Creative Commons Attribution License, which permits unrestricted use, distribution, and reproduction in any medium, provided the original work is properly cited.

This paper presents the vibroimpact dynamics of an in-plane tethered subsatellite caused by sudden braking during deployment or retrieval. The full dynamics of the subsatellite are composed of its free-flight and the instantaneous impacts. At the moment of impact, the reflective angle of the subsatellite is envisioned to be equal to its incident angle such that the impact law is obtained. Then, the stability of the periodic vibroimpacts is analyzed using the composite Poincaré map. Further, the vibroimpact responses that do not exceed a specified region are numerically determined via the cell mapping method.

\section{Introduction}

The vibroimpact phenomena of tethered satellites have been observed in experiments since the first on-orbit launch. In the H-9M-69 and S-520-2 missions, for instance, the deployment process was braked suddenly when the tether was deployed to only $38 \mathrm{~m}$ and $65 \mathrm{~m}$, and then a series of impacts occurred [1]. In the TSS-1 mission, impact-like phenomena were observed when the space tether was jammed unexpectedly by a screw installed at an inappropriate position [2]. Moreover, a similar impact behavior was witnessed in a multiapplication survivable tether (MAST) experiment [3]. Furthermore, numerical simulations have indicated that vibroimpacts occurred at the end of the free deployment of a flexible tethered satellite system, as shown in Figure 1 [4]. Matunaga et al. [5] pointed out that the impact phenomena in a tethered satellite system are caused by a sudden tensioning force in the tether. To avoid the impact due to the slack of the tether, Wen et al. $[6,7]$ designed a nonlinear control law with a positive tension constraint for the deployment. Markeyev [8] analyzed the stability of the vibroimpact oscillation of an ideal tethered particle under a uniform gravitational field in terms of a strict analytical solution of periodic motion.

The aim of this paper is to reveal the impact dynamics of a tethered subsatellite system under tether constraint.
The paper is organized as follows. First, the velocities of the subsatellite before and after impact are obtained. Then, a set of composite Poincaré maps is established. Next, the periodic vibroimpacts and their stability via the composite Poincaré map are analyzed. The cell mapping method is used to determine the parameter regions where the impact occurs. Finally, case studies are numerically examined.

\section{Impact Problem of a Tethered Subsatellite}

An in-plane tethered satellite system orbiting Earth under consideration consists of a mother satellite $M$, a subsatellite $S$, and a flexible tether, as shown in Figure 2. The satellites connected by tether are regarded as particles. The tether can be deployed/retrieved by a deployment/retrieval device mounted on two satellites. The mass of the mother satellite is sufficiently larger than that of the subsatellite, so the motion of the mother satellite is immune to the subsatellite motion. Furthermore, it is assumed that the mother satellite operates in a circular orbit with an angular velocity $\Omega$ around the Earth. To describe the motion of the system, an orbital reference frame $o-x y$ with coordinate origin $o$ in the mother satellite is established, wherein the $x$-axis points are in the opposite direction of the motion of the mother satellite, and 


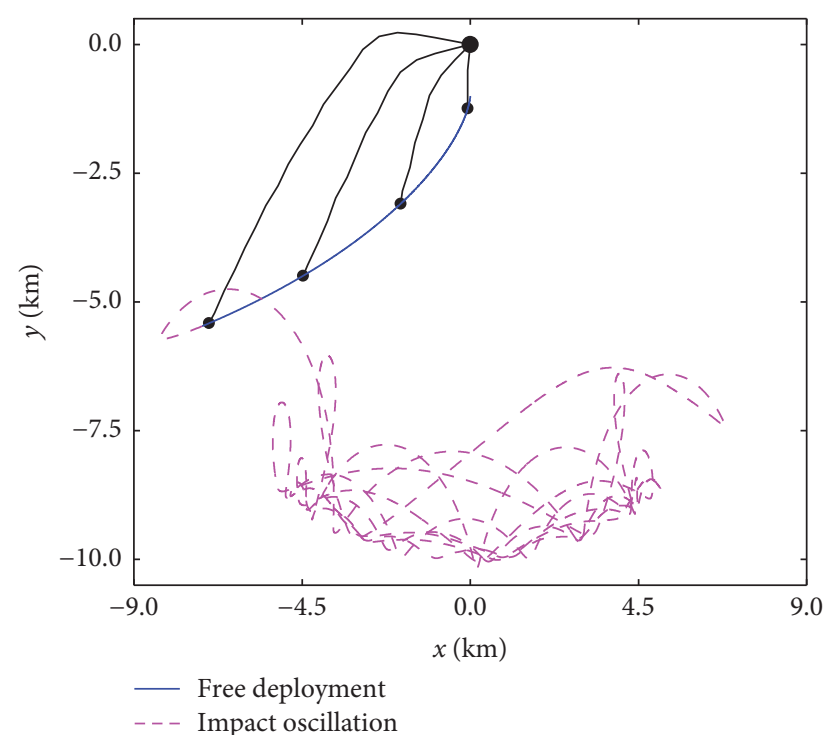

FIGURE 1: Vibroimpacts of a subsatellite [4].

the $y$-axis is on the orbital plane and perpendicular to the $x$ axis.

Provided that the deployment or retrieval process is stopped suddenly at time $t_{k}$ with the tether length $l\left(t_{k}\right)=L$, there follows a subsatellite impact under the tether constraint $C(x, y)=\sqrt{x^{2}+y^{2}}-L=0$, as illustrated in Figure 2 .

The impact is divided into the perfect elastic and inelastic impacts, that is, impact without/with damping due to the elasticity of the tether. As shown in Figure 2, the incident and reflective angles before and after impact are equal to each other; that is, $\varphi_{\text {in }}=\varphi_{\text {re }}$. By letting $x_{1}=x, x_{2}=\dot{x}, x_{3}=y$, and $x_{4}=\dot{y}$, the impact law of the subsatellite can be obtained as follows:

$$
\begin{aligned}
& x_{1}^{+}\left(t_{k}\right)=x_{1}^{-}\left(t_{k}\right) \text {, } \\
& x_{2}^{+}\left(t_{k}\right)=e \cdot\left[\left(\cos ^{2} \theta_{k}-\sin ^{2} \theta_{k}\right) x_{2}^{-}\left(t_{k}\right)\right. \\
& \left.+2 \sin \theta_{k} \cos \theta_{k} x_{4}^{-}\left(t_{k}\right)\right] \text {, } \\
& x_{3}^{+}\left(t_{k}\right)=x_{3}^{-}\left(t_{k}\right) \text {, } \\
& x_{4}^{+}\left(t_{k}\right)=e \cdot\left[2 \sin \theta_{k} \cos \theta_{k} x_{2}^{-}\left(t_{k}\right)\right. \\
& \left.+\left(\sin ^{2} \theta_{k}-\cos ^{2} \theta_{k}\right) x_{4}^{-}\left(t_{k}\right)\right],
\end{aligned}
$$

where the superscripts "-" and “+” represent the moment before and after impact, respectively, and $e$ is the factor of restitution in impact. The pitch angle of the tether at the impact moment $t_{k}$ is

$$
\theta_{k}=\left[\pi-\arccos \frac{x_{3}^{-}\left(t_{k}\right)}{L}\right] \cdot \operatorname{sign}\left[x_{1}^{-}\left(t_{k}\right)\right] .
$$

Equation (1) can be rewritten in the following form:

$$
\begin{aligned}
& \mathbf{x}^{+}\left(t_{k}\right)=\left[\begin{array}{l}
x_{1}^{+}\left(t_{k}\right) \\
x_{2}^{+}\left(t_{k}\right) \\
x_{3}^{+}\left(t_{k}\right) \\
x_{4}^{+}\left(t_{k}\right)
\end{array}\right] \\
& =\left[\begin{array}{cccc}
1 & 0 & 0 & 0 \\
0 & e \cdot\left(\cos ^{2} \theta_{k}-\sin ^{2} \theta_{k}\right) & 0 & e \cdot\left(2 \sin \theta_{k} \cos \theta_{k}\right) \\
0 & 0 & 1 & 0 \\
0 & e \cdot\left(2 \sin \theta_{k} \cos \theta_{k}\right) & 0 & e \cdot\left(\sin ^{2} \theta_{k}-\cos ^{2} \theta_{k}\right)
\end{array}\right]\left[\begin{array}{l}
x_{1}^{-}\left(t_{k}\right) \\
x_{2}^{-}\left(t_{k}\right) \\
x_{3}^{-}\left(t_{k}\right) \\
x_{4}^{-}\left(t_{k}\right)
\end{array}\right] \\
& \triangleq \mathbf{G}\left(\mathbf{x}^{-}\left(t_{k}\right)\right),
\end{aligned}
$$

where $\cos \theta_{k}=-x_{3}^{-}\left(t_{k}\right) / L$ and $\sin \theta_{k}=x_{1}^{-}\left(t_{k}\right) / L$.

After one impact, the subsatellite could freely move without constraint until the beginning of the next impact. For such a free-flight phase, by the straightforward application of Hill's equations, the motion equations of the subsatellite are as follows:

$$
\begin{aligned}
& \dot{x}_{1}=x_{2}, \\
& \dot{x}_{2}=2 \Omega x_{4}, \\
& \dot{x}_{3}=x_{4}, \\
& \dot{x}_{4}=3 \Omega^{2} x_{3}-2 \Omega x_{2},
\end{aligned}
$$

where the dot represents the derivative with respect to time $t$. Equation (4) can be expressed in the following state equation:

$$
\dot{\mathbf{x}}=\mathbf{F}(\mathbf{x}, t)=\mathbf{A} \mathbf{x},
$$

where $\mathbf{x}=\left[\begin{array}{llll}x_{1} & x_{2} & x_{3} & x_{4}\end{array}\right]^{T}$ and

$$
\mathbf{A}=\left[\begin{array}{cccc}
1 & 0 & 0 & 0 \\
0 & 0 & 0 & 2 \Omega \\
0 & 0 & 1 & 0 \\
0 & -2 \Omega & 3 \Omega^{2} & 0
\end{array}\right]
$$

By taking $\mathbf{x}^{+}\left(t_{k}\right)$ as the initial states, the analytical solutions of motion of the subsatellite can be obtained as

$$
\mathbf{x} \triangleq \mathbf{f}\left(\mathbf{x}^{+}\left(t_{k}\right), t\right)=\left[\begin{array}{cccc}
1 & -3 t+\frac{4 \sin \Omega t}{\Omega} & 6(\Omega t-\sin \Omega t) & \frac{2(1-\cos \Omega t)}{\Omega} \\
0 & -3+4 \cos \Omega t & 6 \Omega(1-\cos \Omega t) & 2 \sin \Omega t \\
0 & \frac{2(\cos \Omega t-1)}{\Omega} & -3 \cos \Omega t+4 & \frac{\sin \Omega t}{\Omega} \\
0 & -2 \sin \Omega t & 3 \Omega \sin \Omega t & \cos \Omega t
\end{array}\right]\left[\begin{array}{c}
x_{1}^{+}\left(t_{k}\right) \\
x_{2}^{+}\left(t_{k}\right) \\
x_{3}^{+}\left(t_{k}\right) \\
x_{4}^{+}\left(t_{k}\right)
\end{array}\right]
$$




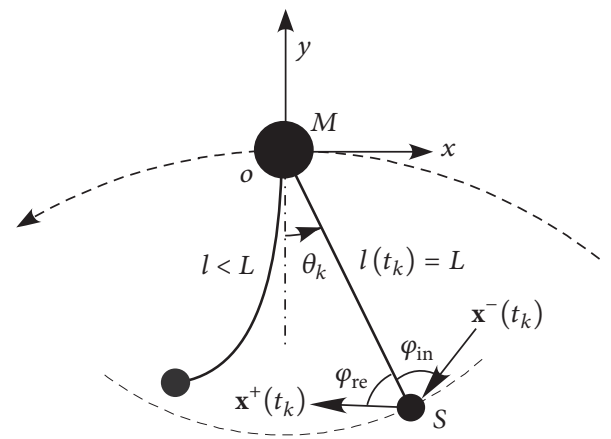

FIGURE 2: Impact process of a tethered subsatellite system.

Equations (3) and (7) give the vibroimpact dynamics of the subsatellite.

\section{Stability of Periodic Vibroimpacts}

The Poincaré map will be used to analyze the stability of the periodic vibroimpacts. The Poincaré section is defined as

$$
\Sigma=\left\{\left(x_{1}, x_{2}, x_{3}, x_{4}\right) C\left(x_{1}, x_{3}\right)=0\right\} .
$$

The full dynamics of the subsatellite are determined by the following two maps:

$$
\begin{gathered}
\mathbf{P}^{f}: \Sigma^{+} \longrightarrow \Sigma^{-}, \\
\mathbf{P}^{i}: \Sigma^{-} \longrightarrow \Sigma^{+},
\end{gathered}
$$

where $\mathbf{P}^{f}$ and $\mathbf{P}^{i}$ represent the maps for the free-flight and impact phases of the subsatellite, respectively. The composite Poincaré map is

$$
\mathbf{P}=\mathbf{P}_{n}^{i} \circ \mathbf{P}_{n}^{f} \circ \cdots \circ \mathbf{P}_{1}^{i} \circ \mathbf{P}_{1}^{f} .
$$

Clearly, the fixed point $\overline{\mathbf{x}}$ of the composite map corresponds to a vibroimpact oscillation of period $\bar{T}$. Taking the Taylor expansion of $\mathbf{P}$ in the vicinity of the fixed point gives the following:

$$
\mathbf{P}(\mathbf{x}, \bar{T})-\mathbf{P}(\overline{\mathbf{x}}, \bar{T})=\mathbf{J}(\overline{\mathbf{x}}, \bar{T})(\mathbf{x}-\overline{\mathbf{x}}),
$$

where $\bar{T}=\sum_{k=1}^{n} \bar{T}_{k}$, and $\bar{T}_{k}$ is the free-flight time of the subsatellite after the $k$ th impact. Based on the perfect elastic impact, $\mathbf{J}(\overline{\mathbf{x}}, \bar{T})$ is the Jacobian of (10) calculated at $\overline{\mathbf{x}}$ using the chain rule; namely,

$$
\mathbf{J}(\overline{\mathbf{x}}, \bar{T})=\mathbf{J}^{i}\left(\mathbf{x}^{-}\left(t_{n}\right)\right) \mathbf{J}^{f}\left(\bar{T}_{n}\right) \cdots \mathbf{J}^{i}\left(\mathbf{x}^{-}\left(t_{1}\right)\right) \mathbf{J}^{f}\left(\bar{T}_{1}\right)
$$

with

$$
\begin{aligned}
& \mathbf{J}^{f}\left(\bar{T}_{k}\right)=\operatorname{Df}\left(\mathbf{x}^{+}\left(t_{k-1}\right), \bar{T}_{k}\right)=\left[\begin{array}{cccc}
1 & -3 \bar{T}_{k}+\frac{4 \sin \Omega \bar{T}_{k}}{\Omega} & 6\left(\Omega \bar{T}_{k}-\sin \Omega \bar{T}_{k}\right) & \frac{2\left(1-\cos \Omega \bar{T}_{k}\right)}{\Omega} \\
0 & -3+4 \cos \Omega \bar{T}_{k} & 6 \Omega\left(1-\cos \Omega \bar{T}_{k}\right) & 2 \sin \Omega \bar{T}_{k} \\
0 & \frac{2\left(\cos \Omega \bar{T}_{k}-1\right)}{\Omega} & -3 \cos \Omega \bar{T}_{k}+4 & \frac{\sin \Omega \bar{T}_{k}}{\Omega} \\
0 & -2 \sin \Omega \bar{T}_{k} & 3 \Omega \sin \Omega \bar{T}_{k} & \cos \Omega \bar{T}_{k}
\end{array}\right], \\
& \mathbf{J}^{i}\left(\mathbf{x}^{-}\left(t_{k}\right)\right)=\mathbf{D G}\left(\mathbf{x}^{-}\left(t_{k}\right)\right)=\left[\begin{array}{cccc}
1 & 0 & 0 & 0 \\
0 & \frac{\left[x_{3}^{-}\left(t_{k}\right)\right]^{2}}{L^{2}}-\frac{\left[x_{1}^{-}\left(t_{k}\right)\right]^{2}}{L^{2}} & 0 & -2 \frac{x_{1}^{-}\left(t_{k}\right) x_{3}^{-}\left(t_{k}\right)}{L^{2}} \\
0 & 0 & 1 & 0 \\
0 & -2 \frac{x_{1}^{-}\left(t_{k}\right) x_{3}^{-}\left(t_{k}\right)}{L^{2}} & 0 & \frac{\left[x_{1}^{-}\left(t_{k}\right)\right]^{2}}{L^{2}}-\frac{\left[x_{3}^{-}\left(t_{k}\right)\right]^{2}}{L^{2}}
\end{array}\right]\left[\begin{array}{l}
x_{1}^{-}\left(t_{k}\right) \\
x_{2}^{-}\left(t_{k}\right) \\
x_{3}^{-}\left(t_{k}\right) \\
x_{4}^{-}\left(t_{k}\right)
\end{array}\right] \\
& =\left[\begin{array}{cccc}
1 & 0 & 0 & 0 \\
\frac{-2\left[x_{1}^{-}\left(t_{k}\right) x_{2}^{-}\left(t_{k}\right)+x_{3}^{-}\left(t_{k}\right) x_{4}^{-}\left(t_{k}\right)\right]}{L^{2}} & \frac{\left[x_{3}^{-}\left(t_{k}\right)\right]^{2}-\left[x_{1}^{-}\left(t_{k}\right)\right]^{2}}{L^{2}} & \frac{2\left[x_{2}^{-}\left(t_{k}\right) x_{3}^{-}\left(t_{k}\right)-x_{1}^{-}\left(t_{k}\right) x_{4}^{-}\left(t_{k}\right)\right]}{L^{2}} & \frac{-2 x_{1}^{-}\left(t_{k}\right) x_{3}^{-}\left(t_{k}\right)}{L^{2}} \\
0 & 0 & 1 & 0 \\
\frac{2\left[x_{1}^{-}\left(t_{k}\right) x_{4}^{-}\left(t_{k}\right)-x_{2}^{-}\left(t_{k}\right) x_{3}^{-}\left(t_{k}\right)\right]}{L^{2}} & \frac{-2 x_{1}^{-}\left(t_{k}\right) x_{3}^{-}\left(t_{k}\right)}{L^{2}} & \frac{-2\left[x_{1}^{-}\left(t_{k}\right) x_{2}^{-}\left(t_{k}\right)+x_{3}^{-}\left(t_{k}\right) x_{4}^{-}\left(t_{k}\right)\right]}{L^{2}} & \frac{\left[x_{1}^{-}\left(t_{k}\right)\right]^{2}-\left[x_{3}^{-}\left(t_{k}\right)\right]^{2}}{L^{2}}
\end{array}\right] .
\end{aligned}
$$




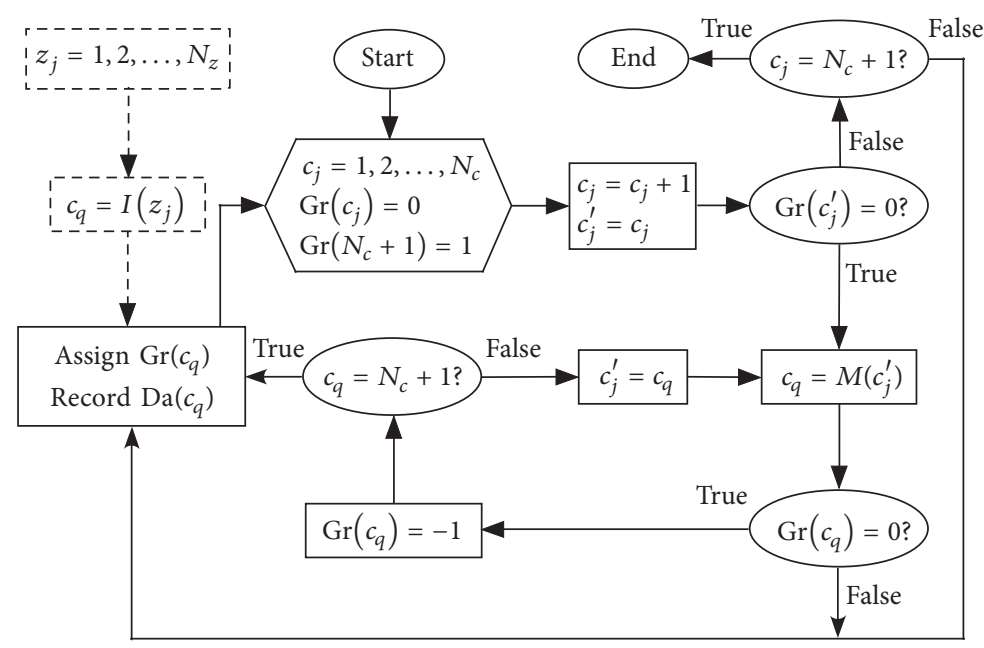

FIGURE 3: Flow chart of the cell mapping algorithm.

Thus, the stability of the periodic vibroimpacts can be determined by the roots of the Jacobian $\mathbf{J}(\overline{\mathbf{x}}, \bar{T}), \lambda_{j}(j=$ $1,2,3,4)$; that is,

$$
\begin{aligned}
& \left|\lambda_{j}\right|_{\max }<1 \quad \text { asymptotically stable, } \\
& \left|\lambda_{j}\right|_{\max }>1 \quad \text { unstable, } \\
& \left|\lambda_{j}\right|_{\max }=1 \quad \text { undetermined. }
\end{aligned}
$$

\section{Algorithm of Parameter Domain}

To obtain an insight into the vibrodynamics of a tethered system, the parameter domain state not exceeding a specified region $\Pi$ is calculated using the cell mapping method [9]. The specified region $\Pi$ is also a system state in an expected pitch angle or radial impact velocity range of the subsatellite. According to the polar coordinate system shown in Figure 2,

$$
\begin{aligned}
& x=l \sin \theta, \\
& y=-l \cos \theta .
\end{aligned}
$$

The study on the parameter domain is divided into two parts. First, the three-dimensional state space is divided into $N_{c}=n_{\theta}^{i} n_{\dot{\theta}}^{i} n_{j}^{i}$ cells as $c_{j}\left(j=1,2, \ldots, N_{c}\right)$ with $n_{\theta}^{i}, n_{\dot{\theta}}^{i}$, and $n_{i}^{i}$ elements in $\theta, \dot{\theta}$, and $\dot{l}$. The sink cell is defined as $c_{N_{c}+1}=N_{c}+1$. The minute flow chart of the cell mapping algorithm is given in Figure 3, where the group numbers $\operatorname{Gr}\left(c_{q}\right)$ are assigned for cell sequences $\left\{c_{q}\right\}$, and the state information $\operatorname{Da}\left(c_{q}\right)$ is recorded, so that the maximum value for each cell mapping can be obtained based on $\operatorname{Gr}\left(c_{q}\right)$ and $\mathrm{Da}\left(c_{q}\right)$. After completion of the calculation, the states of all cells can be analyzed. The corresponding cell will be not in the domain of attraction (i.e., the parameter domain) as long as the maximum of any mapping cell of the cell exceeds the specified region $\Pi$. Hence, the domain of attraction is dependent upon the attractor, all cells of which are maintained in the specified region $\Pi$.
To extend the application to all system states $(\theta, \dot{\theta}, l, \dot{l})$, a similar flow process of the cell mapping method is conducted. The ranges $\left[\theta_{\min }, \theta_{\max }\right],\left[\dot{\theta}_{\min }, \dot{\theta}_{\max }\right],\left[l_{\min }, l_{\max }\right]$, and $\left[i_{\min }, \dot{l}_{\max }\right]$ are uniformly divided into $n_{\theta}, n_{\dot{\theta}}, n_{l}$, and $n_{i}$ elements. Then, the state space of the system is divided into $N_{z}=n_{\theta} n_{\dot{\theta}} n_{l} n_{i}$ cells as $z_{j}\left(j=1,2, \ldots, N_{z}\right)$. Similarly, the sink cell is defined as $z_{N_{z}+1}=N_{z}+1$. For each cell, $z_{j}\left(j=1,2, \ldots, N_{z}\right)$, the corresponding cell $c_{q}=I\left(z_{j}\right)$ can be computed by (7), as shown in the dashed box of Figure 3 . Finally, the parameter domain for the cells of all system states is acquired via $\operatorname{Gr}\left(c_{q}\right)$ and $\mathrm{Da}\left(c_{q}\right)$.

\section{Case Studies}

5.1. Nonlinear Dynamics. Suppose that the tethered system moves in a circular orbit at an altitude of $300 \mathrm{~km}$ with an orbital angular velocity $\Omega=1.1591 \times 10^{-3} \mathrm{rad} / \mathrm{s}$. The total tether length $L=10 \mathrm{~km}$, and $e=1$.

The initial velocity is set as $\left[x_{20}^{+}, x_{40}^{+}\right]^{T}=[-10 \mathrm{~m} / \mathrm{s}, 10 \mathrm{~m} /$ $\mathrm{s}]^{T}$. The bifurcation responses of the pitch angle in Figure 4(a) show that the pitch angle for the impact moment increases with an increase of $x_{10}^{+}$. The pitch angle will particularly exist throughout $[-\pi, \pi]$ once the abscissa $x_{10}^{+}>7113 \mathrm{~m}$. Figure 4(b) shows the bifurcation for $x_{40}^{+}=2 \mathrm{~m} / \mathrm{s}$, in which the critical value of the impact point's coordinate is $(8627 \mathrm{~m},-5057 \mathrm{~m})$. To elaborate the relationship between the coordinate position and the sudden change, the mechanical energy $E$ of the subsatellite in the orbital frame is introduced [10]

$$
\begin{aligned}
E= & V+T \\
= & -\mu_{E} \frac{m_{S}}{R_{E}}+\frac{\mu_{E} m_{S} L^{2}}{2 R_{E}^{3}}\left[1-3\left(\frac{x_{30}^{+}}{L}\right)^{2}\right] \\
& +\frac{1}{2} m_{S}\left(x_{20}^{+2}+x_{40}^{+2}\right),
\end{aligned}
$$




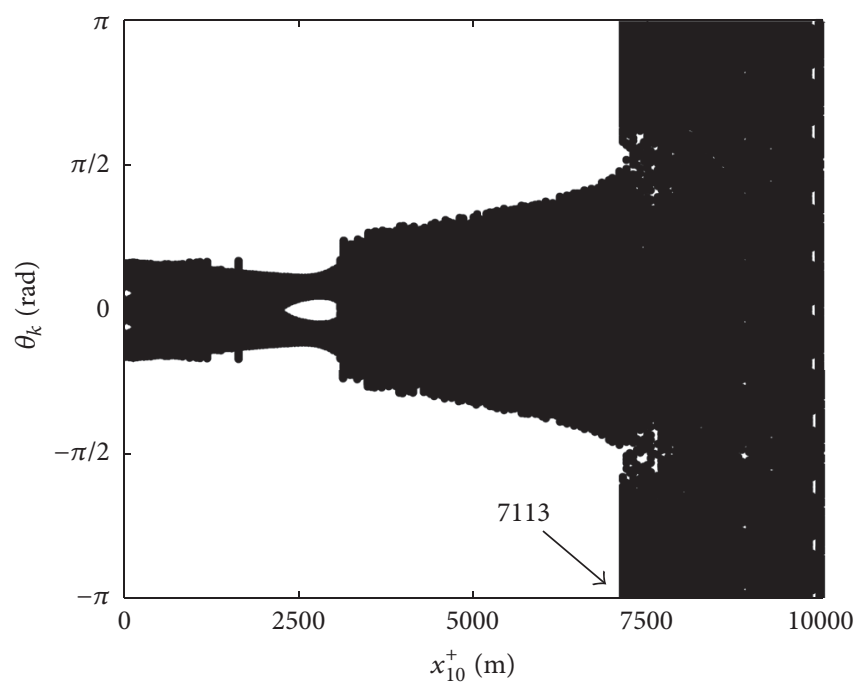

(a) $x_{40}^{+}=10 \mathrm{~m} / \mathrm{s}$

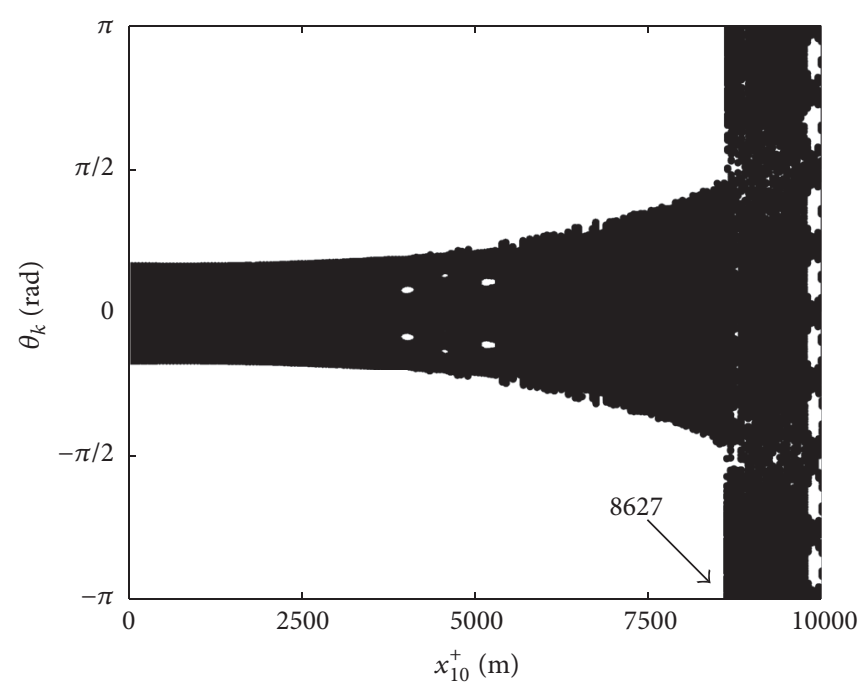

(b) $x_{40}^{+}=2 \mathrm{~m} / \mathrm{s}$

FIGURE 4: Bifurcations of pitch angles with increase of $x_{10}^{+}$.

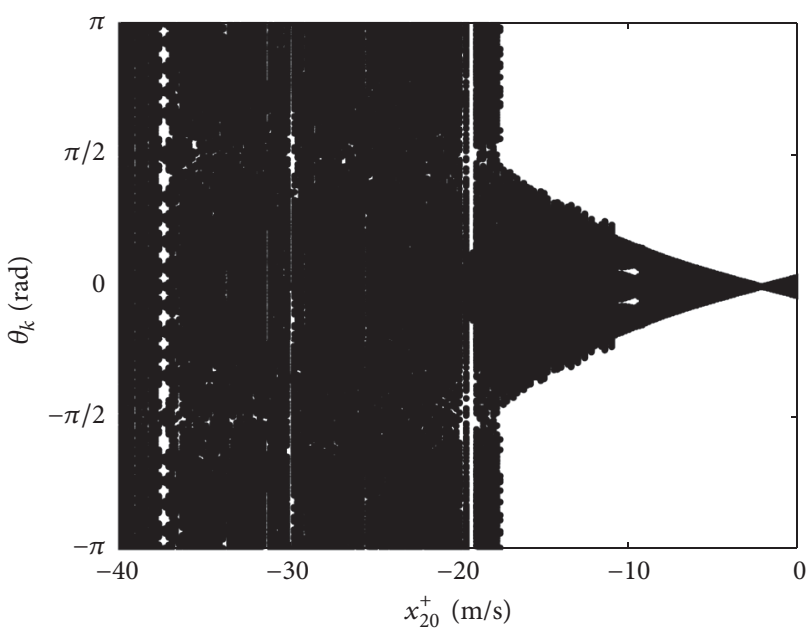

(a) $x_{40}^{+}=10 \mathrm{~m} / \mathrm{s}$

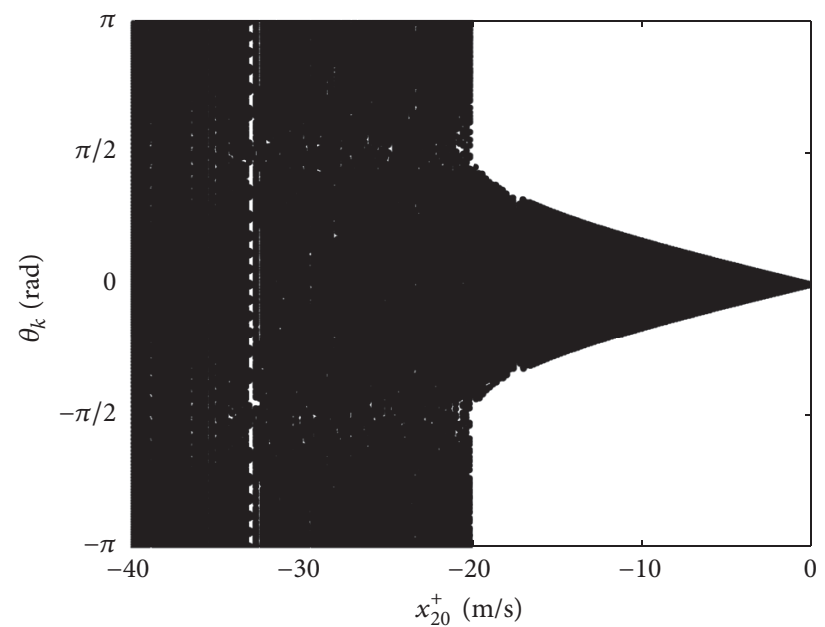

(b) $x_{40}^{+}=2 \mathrm{~m} / \mathrm{s}$

FIGURE 5: Bifurcations of pitch angle with increase of $x_{20}^{+}$.

where $\mu_{E}=3.986 \times 10^{14} \mathrm{~m}^{3} / \mathrm{s}^{2}$ is the gravitational parameter of the Earth, $R_{E}=6371 \mathrm{~km}$ is the mean radius of the Earth, and $m_{S}=50 \mathrm{~kg}$ is the mass of the subsatellite. The critical values $E_{0 c}$ of the initial energy are $-2.987554706 \times 10^{9} \mathrm{~J} \pm$ $10 \mathrm{~J}$. The phenomenon that the pitch angle is throughout $[-\pi, \pi]$ will appear when the initial energy of the system is larger than $E_{0 c}$.

Figure 5 gives the bifurcation responses of the pitch angle when the initial positions $\left(x_{10}^{+}, x_{30}^{+}\right)$are $(0,-L)$. Figure 5 shows that the amplitudes of pitch angle $\theta_{k}$ decrease suddenly if the initial velocities $x_{20}^{+}>-17.44 \mathrm{~m} / \mathrm{s}$ or $x_{20}^{+}>-20.00 \mathrm{~m} / \mathrm{s}$. Both the absolute values of the critical velocities can be calculated as approximately $\sqrt{\left(x_{20}^{+}\right)^{2}+\left(x_{40}^{+}\right)^{2}}=20.10 \mathrm{~m} / \mathrm{s}$, and the critical energy is in accord with only $E_{0 c}$. Consequently, it can be concluded that the initial energy $E_{0}$ plays an important role in the impact dynamics of the tethered satellite system.
For the above-determined system parameters, the periodic, quasi-periodic, and irregular motions will appear as the initial energy changes. For $x_{10}^{+}=0, x_{20}^{+}=-2.12496 \mathrm{~m} / \mathrm{s}$, $x_{30}^{+}=-L$, and $x_{40}^{+}=10 \mathrm{~m} / \mathrm{s}$, for example, a periodic motion is exhibited in Figure 6. Figure 6(a) plots the motion trajectory of the subsatellite in the orbital frame $o-x y$. As shown in Figure 6(a), the periodic motion is constituted by one free-flight and one impact. The subsatellite flies with only the Coriolis force acting along the solid curve and comes back to the initial impact position $(0,-L)$ for $561 \mathrm{~s}$. Figure 6(b) gives the coordinate positions of 1000 impacts. For a clearer observation, the abscissas of the impacts are presented in Figure 6(c). The abscissas converge within the range of $(-0.03 \mathrm{~m}, 0.03 \mathrm{~m})$. The phase points on $C(\mathbf{x})$ are drawn in Figure $6(\mathrm{~d})$. The converging phase points indicate that this is a periodic motion with one impact. 


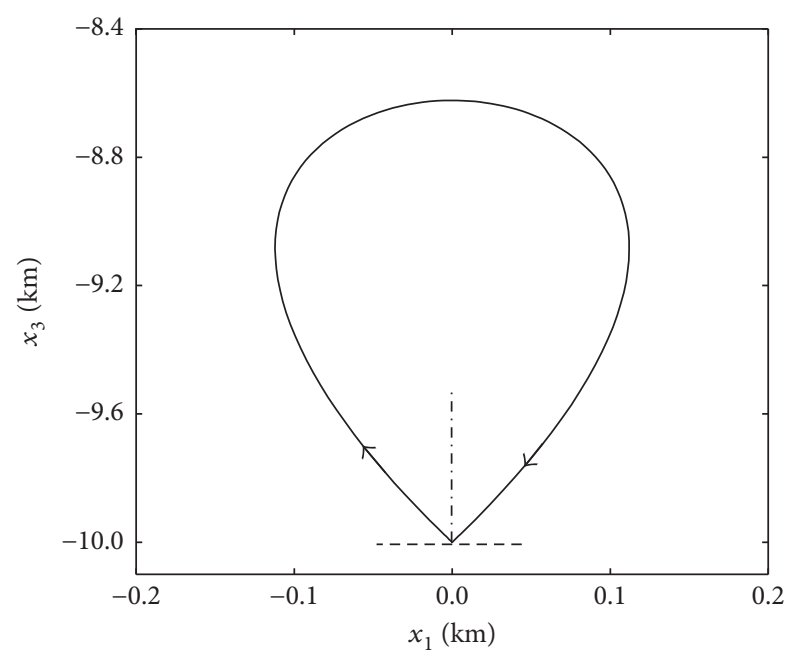

(a) Trajectory of the subsatellite in the orbital frame

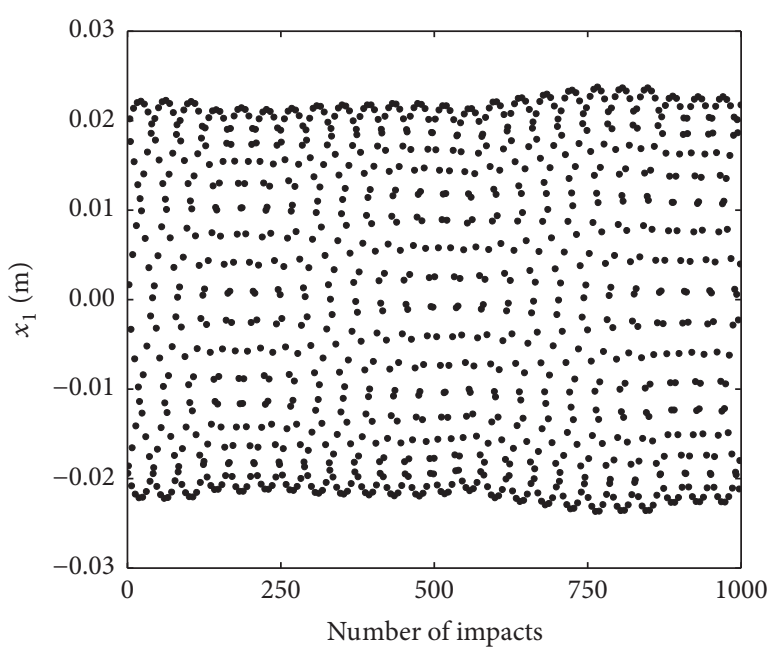

(c) Change of $x_{1}$ coordinate of impacts

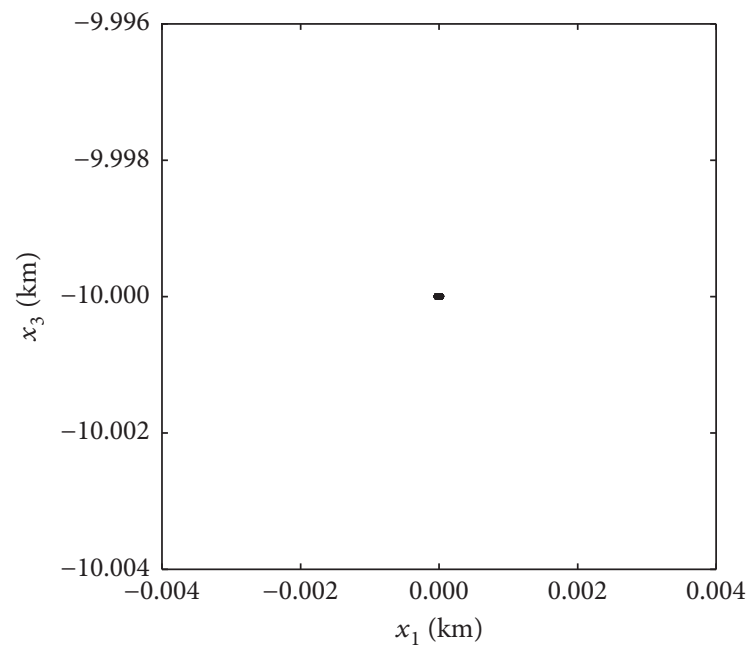

(b) Position coordinates of impacts

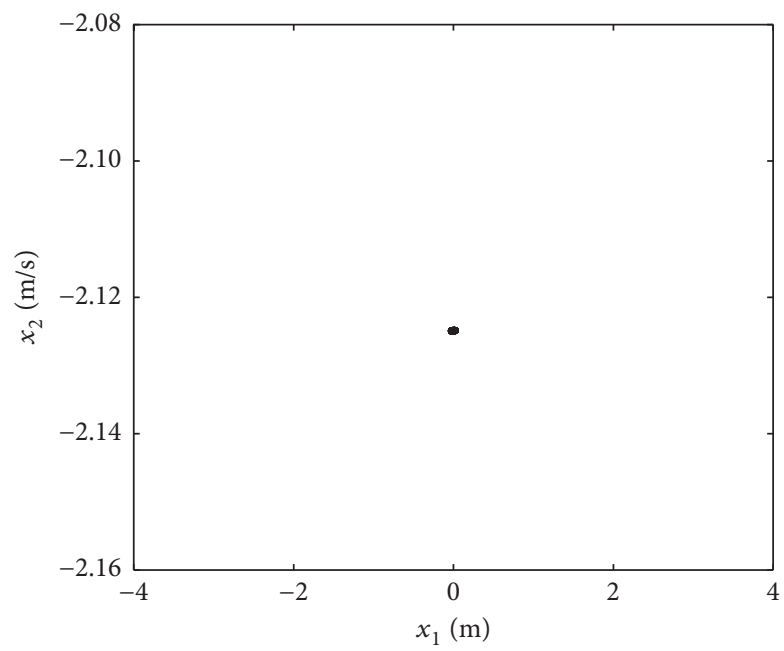

(d) Phase points on $C(\mathbf{x})$

FIgURE 6: Periodic motion with one impact.

The stability of the periodic motion is studied via the Poincaré map in (12). The eigenvalues of the Jacobian can be calculated as $\lambda_{1}=0.5360, \lambda_{2,3}=0.5202 \pm 0.9136 i$, and $\lambda_{4}=-1.6880$, with $\left|\lambda_{1}\right|=0.5360,\left|\lambda_{2,3}\right|=1.0513$, and $\left|\lambda_{4}\right|=1.6880$. This means that the periodic motion with one impact is unstable. With the initial state of $[0 \mathrm{~m},-2.2 \mathrm{~m} /$ $\mathrm{s},-L, 9.98376 \mathrm{~m} / \mathrm{s}]^{T}$, the impact positions are located on a segment of $\mathrm{C}(\mathbf{x})$, and the phase trajectory is a closed curve, as shown in Figure 7.

A periodic motion with four impacts is depicted in Figure 8. Figure 8(a) presents the flight trajectory of the subsatellite. As seen in Figure 8(a), starting with the initial point 0 , the motion of the subsatellite without the tether constraint will impact at points 1,2 , and 3 and return to the origin. Figure $8(\mathrm{~b})$ shows the coordinate position of the impacts. The change of state $x_{2}$ with each impact is displayed in Figure $8(\mathrm{c})$. The phase trajectory on $C(\mathbf{x})$ is shown in Figure $8(\mathrm{~d})$, where the four converging points correspond to the states of four impacts. Similarly, the roots of the Jacobian of the periodic motion can be obtained as $\lambda_{1}=0.0284$, $\lambda_{2}=-0.0981, \lambda_{3}=10.8714$, and $\lambda_{4}=-32.9549$. Clearly, $\left|\lambda_{3,4}\right|>1$ means that the periodic motion is unstable.

A quasi-periodic motion is shown in Figure 9. With the initial state $[0 \mathrm{~m},-5 \mathrm{~m} / \mathrm{s},-L, 10 \mathrm{~m} / \mathrm{s}]^{T}$, Figure 9 (a) depicts the coordinate positions of the impacts. The impacts are all situated on a segment of $C(\mathbf{x})$, and Figure 9(b) shows the phase points on $C(\mathbf{x})$. As observed from Figure 9(b), the phase points constitute a closed curve, which concludes that this is a quasi-periodic motion.

Irregular motion will occur if the initial mechanical energy exceeds the critical value $E_{0 c}$. With the assumption of the initial state $[0 \mathrm{~m},-20 \mathrm{~m} / \mathrm{s},-L, 10 \mathrm{~m} / \mathrm{s}]^{T}$, an irregular motion is presented in Figure 10. The coordinate positions of the impacts are distributed densely on the entire $C(\mathbf{x})$, as shown in Figure 10(a). Figure 10(b) sketches the distribution of the phase points on $C(\mathbf{x})$. Evidently, an oscillation with such a wide range caused by the irregular motion might cause serious disaster to other spacecraft in the neighborhood of the tethered system. 


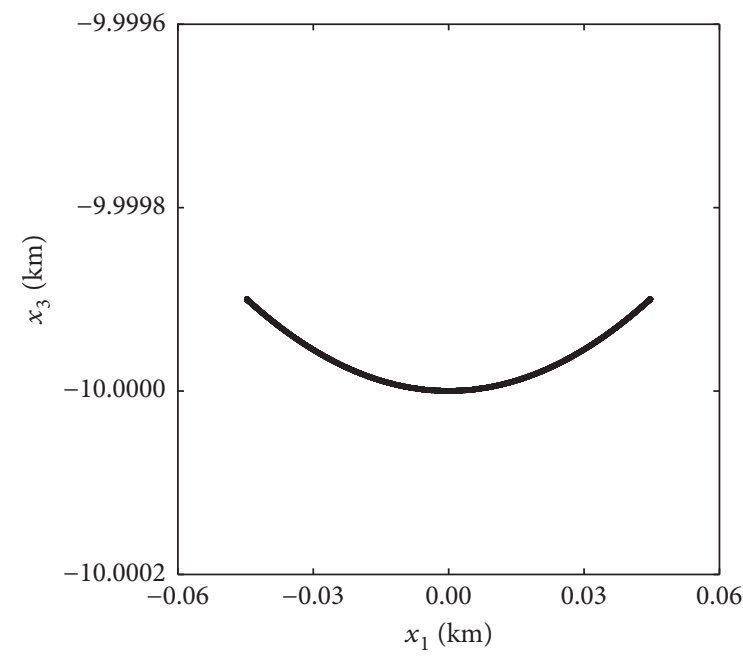

(a) Impact position coordinates

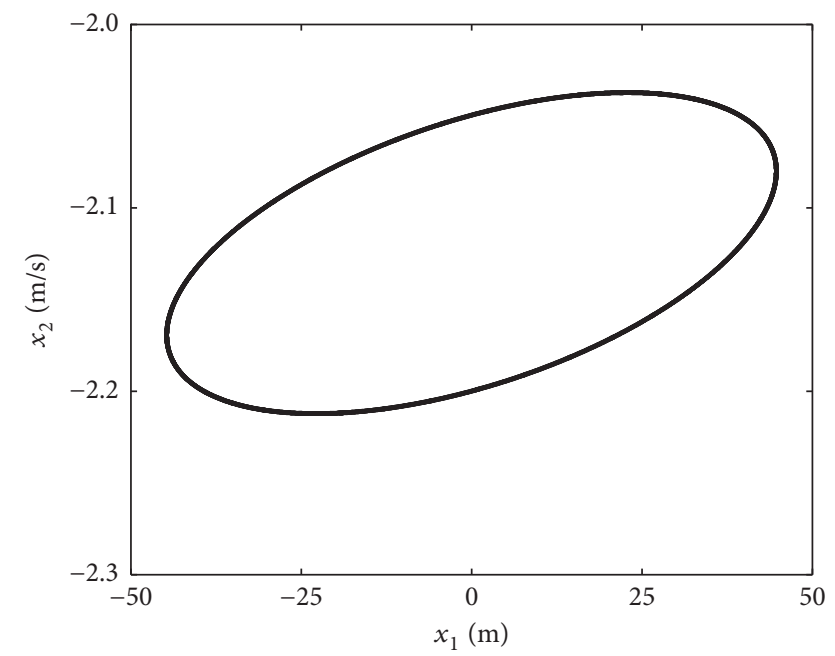

(b) Phase trajectory on $C(\mathbf{x})$

FIgURE 7: Unstable impact motion.

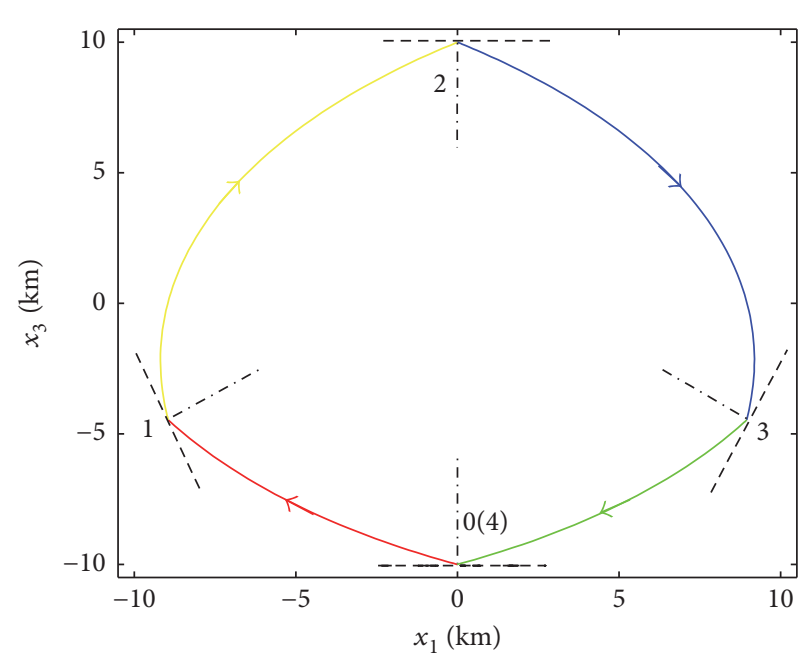

(a) Trajectory of the subsatellite

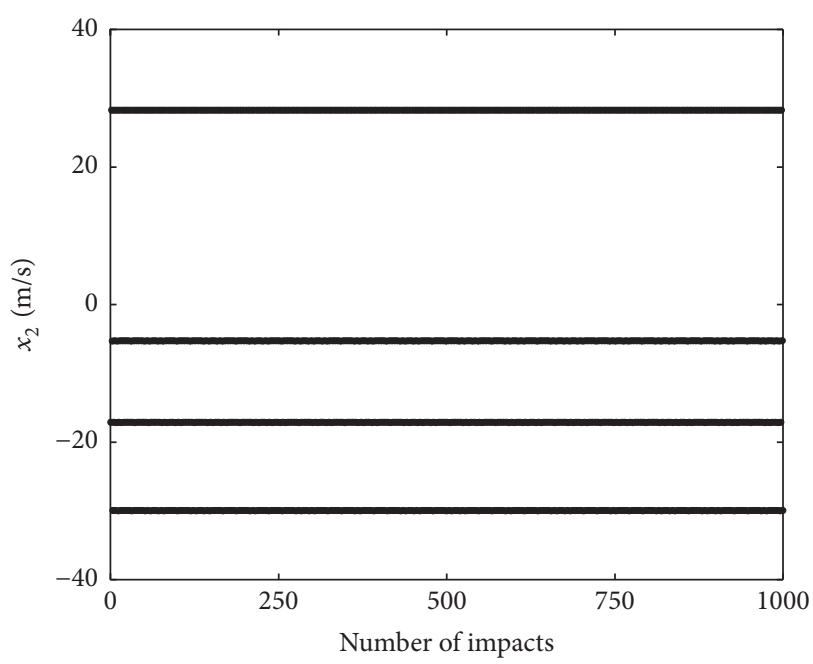

(c) Change of $x_{2}$ of impacts

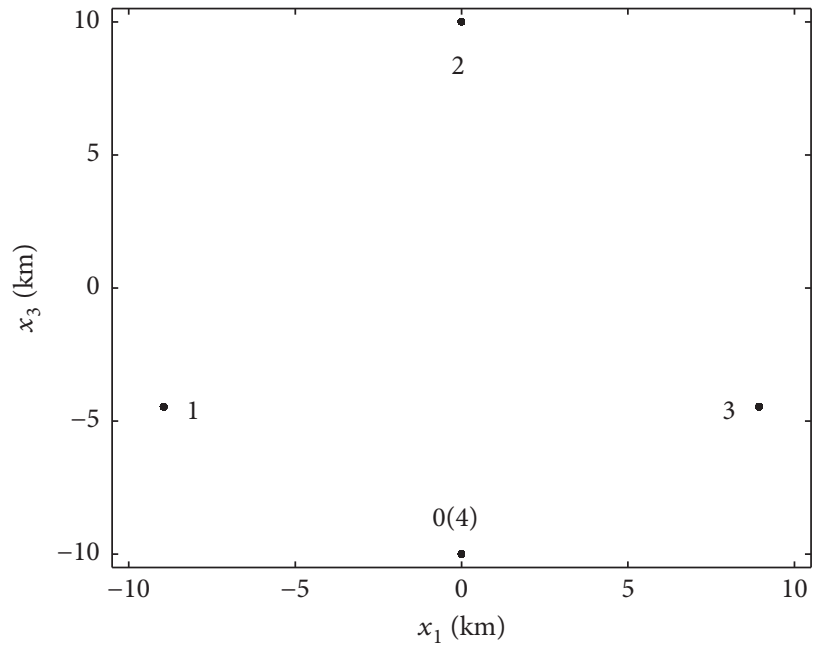

(b) Position coordinates of impacts

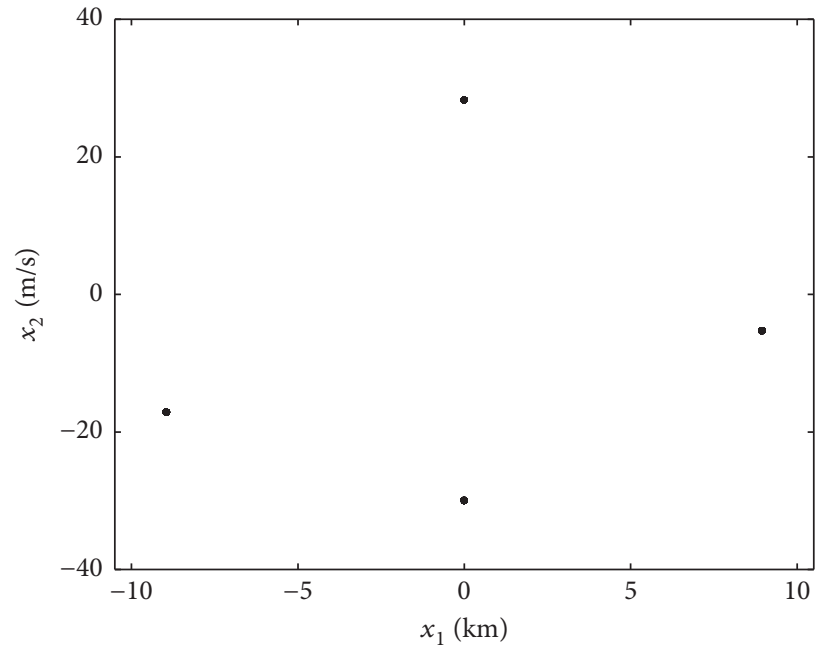

(d) Phase trajectory on $C(\mathbf{x})$

FIgURE 8: Periodic motion with four impacts. 


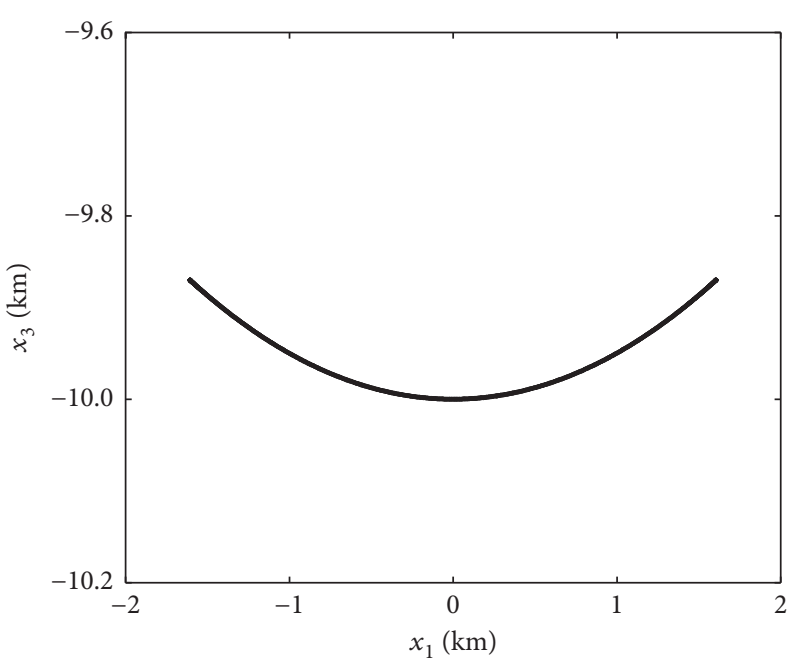

(a) Position coordinates of impacts

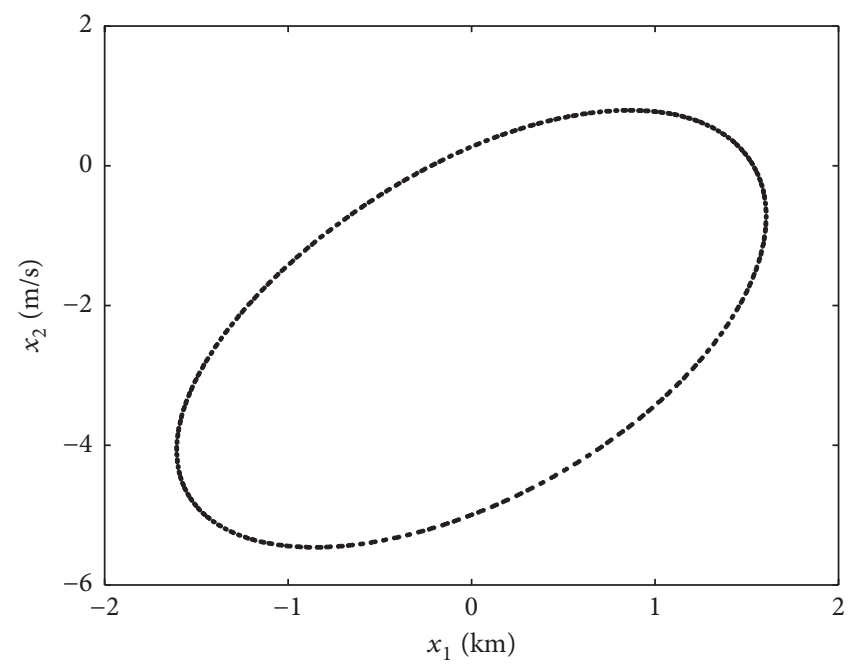

(b) Phase trajectory on $C(\mathbf{x})$

FIgURE 9: Quasi-periodic motion.

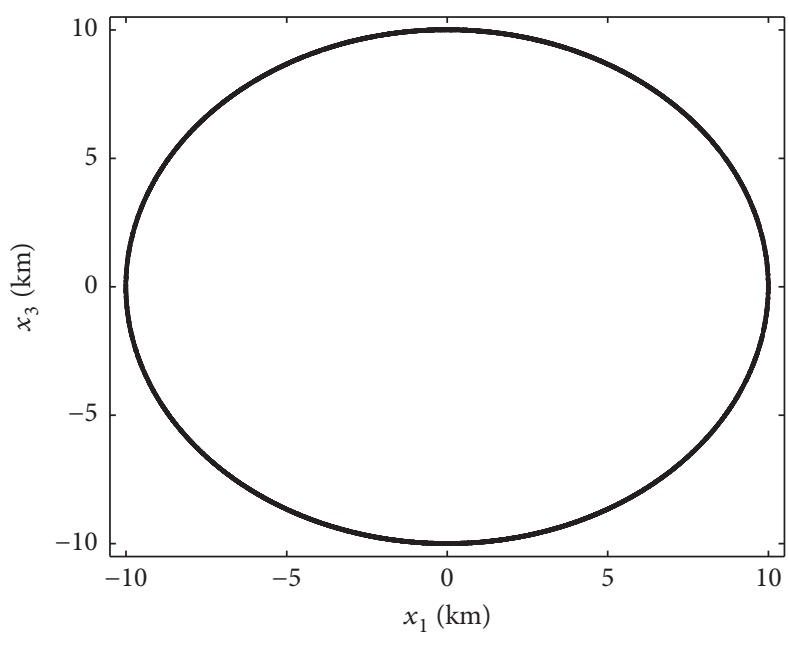

(a) Position coordinates of impacts

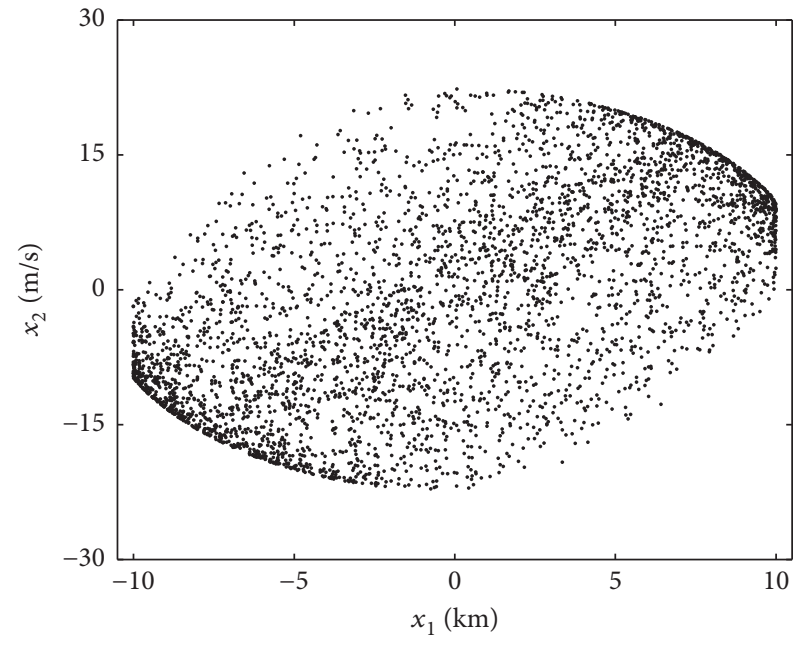

(b) Phase trajectory on $C(\mathbf{x})$

Figure 10: Irregular motions.

In terms of the above irregular motion, a numerical case for the impacts with damping is given in Figure 11. The recovery factor in the impact was set as $e=0.999$; that is, there exists energy dissipation of $0.1 \%$ for each impact. The change of $x_{1}$ coordinate in each impact is presented in Figure 11(a). The corresponding phase trajectory on $C(\mathbf{x})$ is drawn in Figure 11(b). It can be observed from Figure 11(b) that the impact points eventually converge to origin $(0,0)$.

5.2. Parameter Domain. The parameter domain is analyzed through the cell mapping method. First, the state space of impacts on $C$ in the polar coordinate system is discussed. The study areas of the states are $\theta \in[-\pi / 2, \pi / 2], \dot{\theta} \epsilon$ $\left[-10^{-3} \mathrm{rad} / \mathrm{s}, 10^{-3} \mathrm{rad} / \mathrm{s}\right]$, and $i \in[0,18 \mathrm{~m} / \mathrm{s}]$, and the state variables are uniformly divided into 120,120 , and 90 elements, respectively. Thus, the state space of the system on $C$ has 1296000 three-dimensional cells. Then, the corresponding parameter domain (i.e., domain of attraction) can be calculated provided that the specified region (i.e., attractor) is given.

For the specified region $\Pi=\{(\theta, \dot{\theta}, l, \dot{l}) \mid \theta \in[-\pi /$ $6, \pi / 6], l=L\}$, the effect of the radial impact velocity is as shown in Figure 12. As seen from Figure 12, the section area $\theta-\dot{\theta}$ of the parameter domain decreases as $\dot{l}$ increases. Therefore, the expansion of the range of pitch angle with the initial kinetic energy increase is in accordance with the above statement on the initial system energy. For $\dot{l}=6 \mathrm{~m} / \mathrm{s}$, a comparison of the parameter domain with different specified regions is presented in Figure 13, where the specified regions are $\Pi_{1}=\{(\theta, \dot{\theta}, l, \dot{l}) \mid \theta \in[-\pi / 6, \pi / 6], l=L\}, \Pi_{2}=$ $\{(\theta, \dot{\theta}, l, \dot{l}) \mid \theta \in[-\pi / 8, \pi / 8], l=L\}$, and $\Pi_{3}=\{(\theta, \dot{\theta}, l, \dot{l}) \mid$ $\theta \in[-\pi / 12, \pi / 12], l=L\}$. The section area of the parameter domain decreases with the decrease of the specified region. 


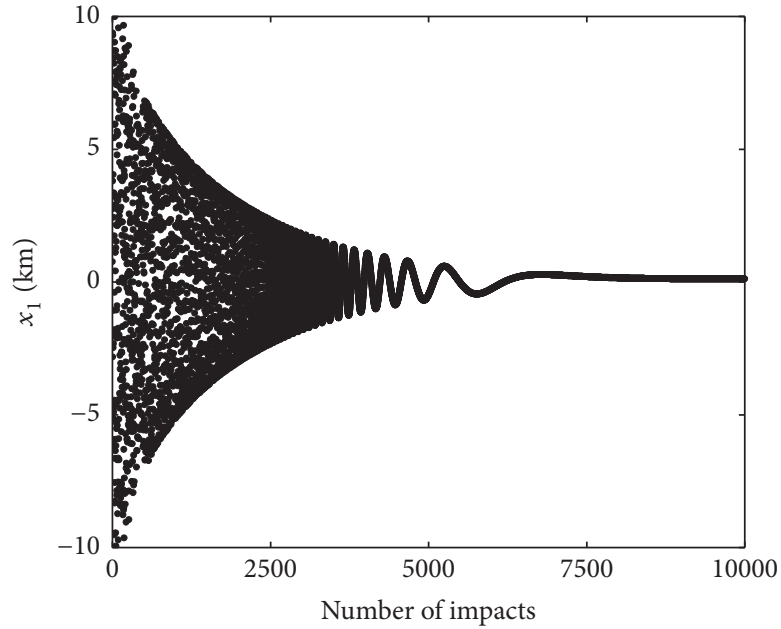

(a) Change of $x_{1}$ coordinate of impacts

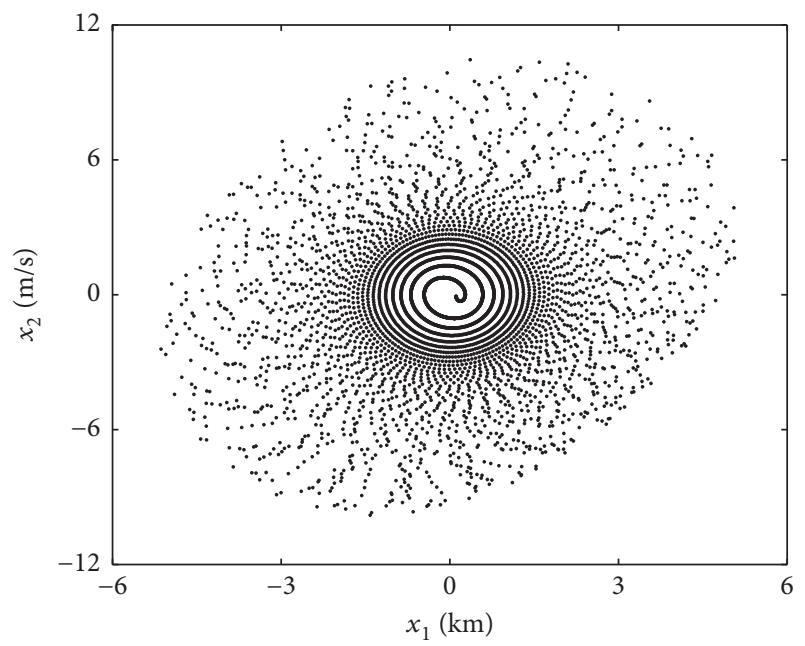

(b) Phase trajectory on $C(\mathbf{x})$

FIGURE 11: Irregular motions with damping factor.

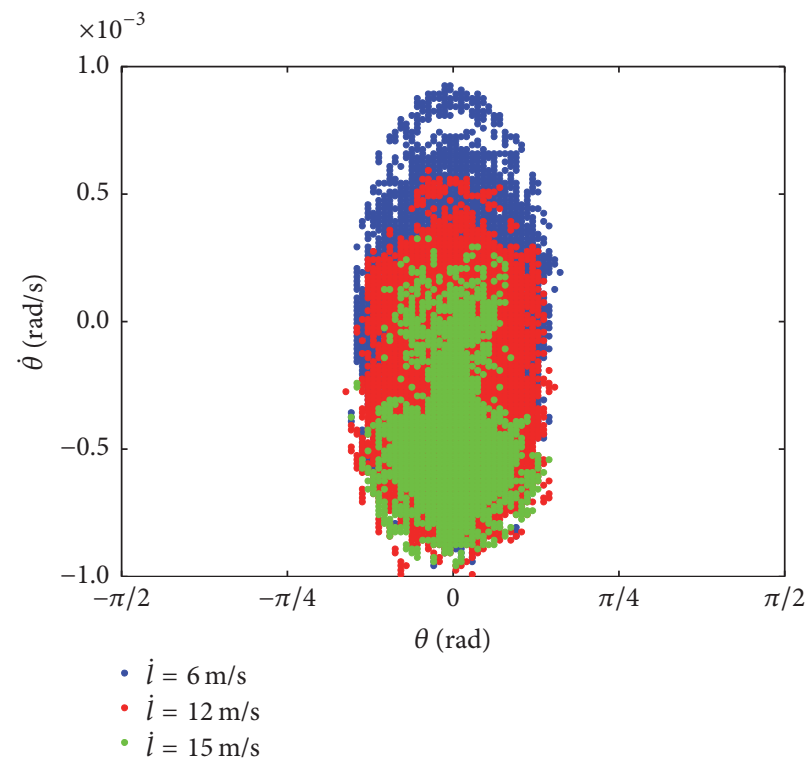

FIGURE 12: Effect of radial impact velocity on the parameter domain.

The influence of the tangential velocity $\dot{\theta}$ on the parameter domain is shown in Figure 14. Figure 14 shows that the maximal section area of the parameter domain appears when $\dot{\theta}=-5 \times 10^{-4} \mathrm{rad} / \mathrm{s}$ because the flight of the subsatellite after impact is towards the positive direction of $\dot{\theta}$ under the Coriolis force. The effect of pitch angle on the parameter domain is provided in Figure 15.

Considering the deployment/retrieval process of the tether before its first impact, the tether length variable $l$ is introduced. The parameter domain on all the system states (i.e., $\theta, \dot{\theta}, l$, and $i$ ) is proposed using the above results for the domain on $C$. The study areas of the pitch angle and pitch angular velocity are $\theta \in[-\pi / 2, \pi / 2]$ and $\dot{\theta} \in\left[-10^{-3} \mathrm{rad} / \mathrm{s}, 10^{-3} \mathrm{rad} / \mathrm{s}\right]$, respectively, and both are divided into 120 elements. Moreover, the ranges of the radial position and radial velocity are $l \in[L / 20, L]$ and $i \in[-18 \mathrm{~m} / \mathrm{s}, 18 \mathrm{~m} / \mathrm{s}]$, respectively, both of which are divided into 30 elements. Accordingly, the state space of the subsatellite is divided into 12960000 four-dimensional cells.

The parameter domain determined by the specified region $\Pi=\{(\theta, \dot{\theta}, l, \dot{l}) \mid \theta \in[-\pi / 6, \pi / 6], l=L\}$ is depicted in Figure 16. For all of the state cells with an initial radial position $l_{0}>8 \mathrm{~km}$ and an initial radial velocity $\dot{l}_{0}=0.6 \mathrm{~m} / \mathrm{s}$, the parameter domain corresponds to the blue zone in Figures 16(a) and 16(b). These figures show that the contrasted red zone decreases as $l_{0}$ decreases or $i_{0}$ increases.

In addition, it is important to study the specified region based on the radial impact velocity. The break of the space 


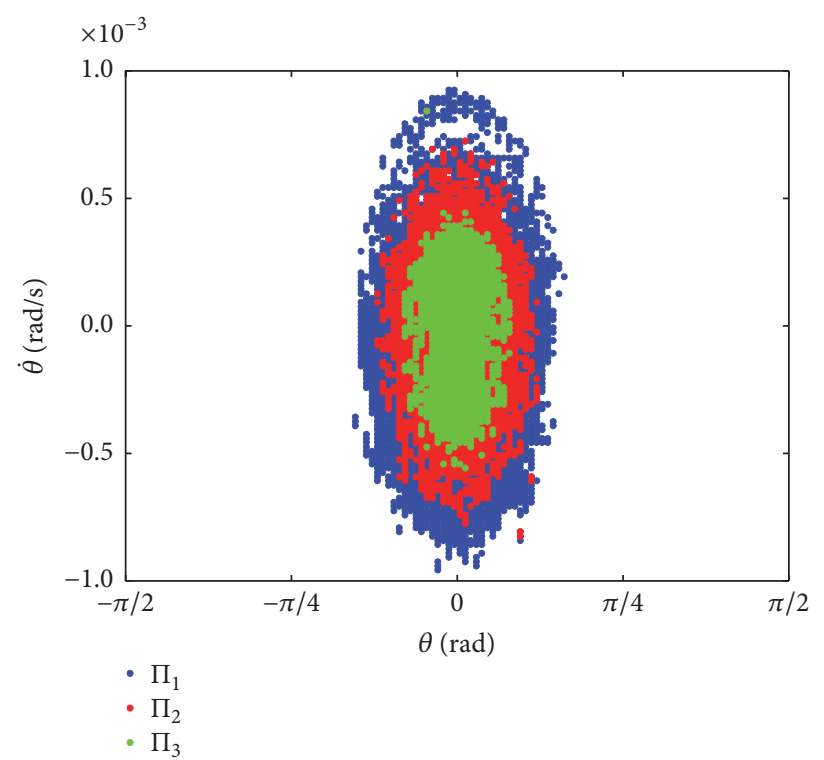

FIGURE 13: Comparison of parameter domains based on different specified regions.

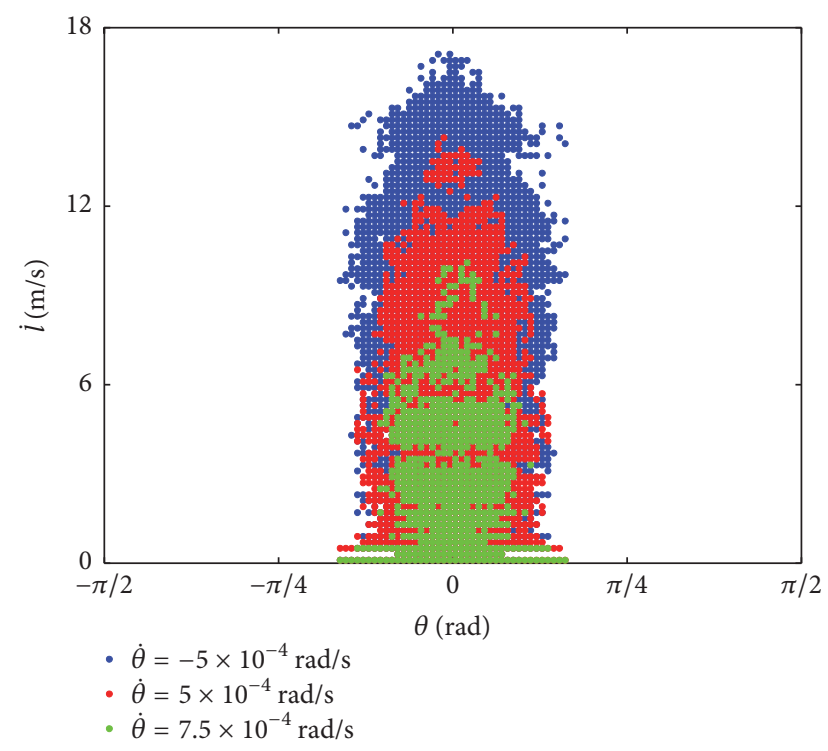

FIGURE 14: Effect of tangential velocity on the parameter domain.

tether will occur once the tether cannot undergo the impact force dependent upon the impact velocity, which might cause more serious damage. The parameter domain governed by the specified region $\Pi=\{(\theta, \dot{\theta}, l, \dot{l}) \mid l=L, \dot{l}<15 \mathrm{~m} / \mathrm{s}\}$ is drawn in Figure 16. Let initial radial velocity $i_{0}=0.6 \mathrm{~m} / \mathrm{s}$, so the effect of the initial radial position on the parameter domain is presented in Figure 17(a). With the assumption of the initial radial position $l_{0}>8 \mathrm{~km}$, the effect of the initial radial velocity on the parameter domain is shown in Figure 17(b). The consequence of the numerical simulations indicates that the parameter domain decreases with the decrease of $l_{0}$ or with the increase of $i_{0}$.

Once the system state cell is inside a parameter domain, the state of the system will be retained in a specified region

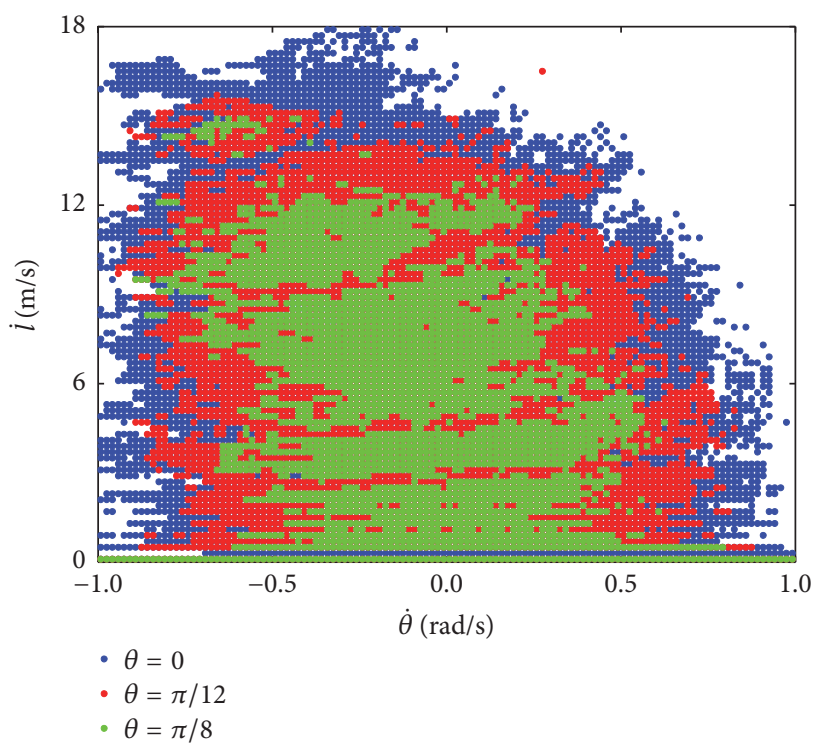

FIGURE 15: Effect of pitch angle on the parameter domain.

even if the tether loses control abruptly. Hence, the parameter domain can be treated as a constraint condition to design the tether deployment/retrieval control law, which is capable of maintaining the subsatellite in an expected region.

\section{Conclusions}

An impact law of an in-plane tethered subsatellite system is given. The periodic vibroimpact motions are investigated using the composite Poincare map. The periodic, quasiperiodic, and irregular vibroimpacts such as one impact or four impacts are found. A parameter domain is proposed to 


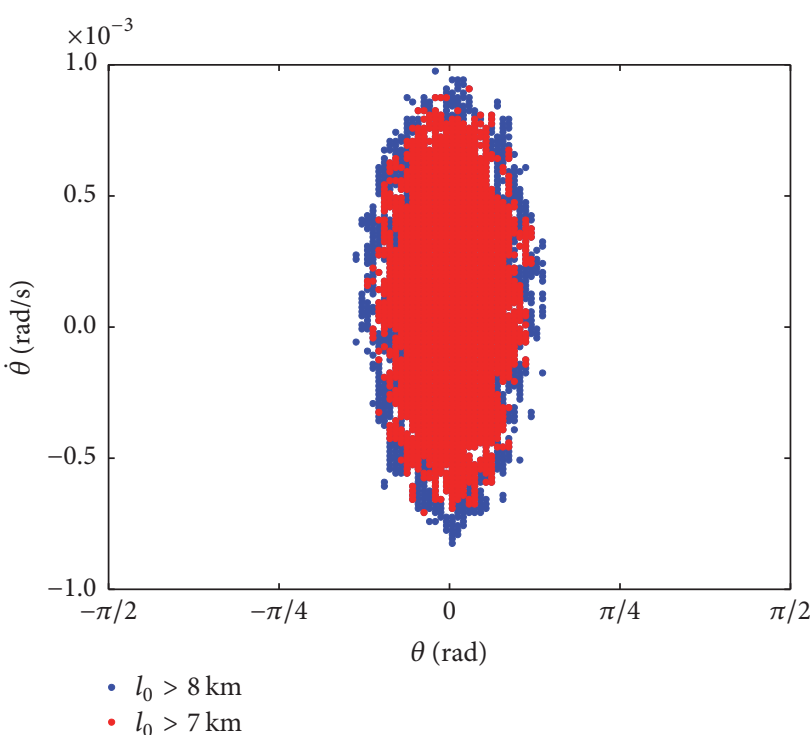

(a) Effect of initial radial position of subsatellite on parameter domain

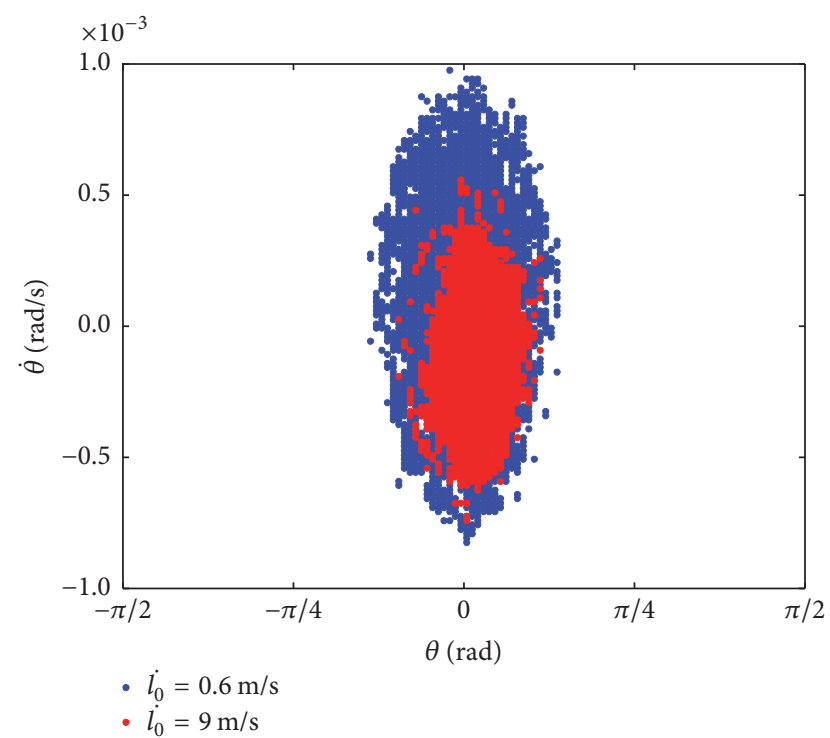

(b) Effect of initial radial velocity of subsatellite on parameter domain FIGURE 16: Dependence of parameter domain in the range $\theta \in[-\pi / 6, \pi / 6]$.

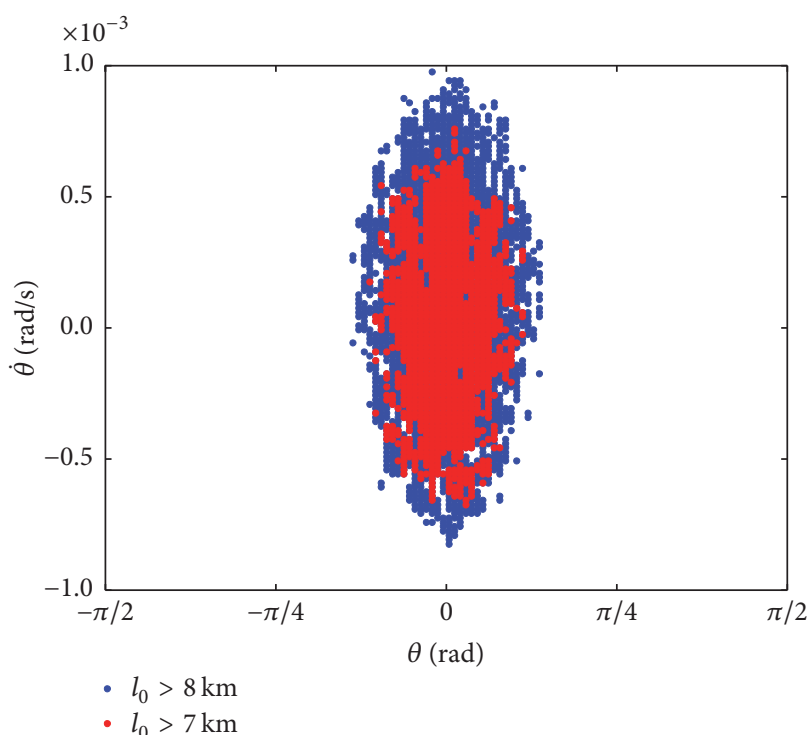

(a) Effect of initial radial position of subsatellite on the parameter domain

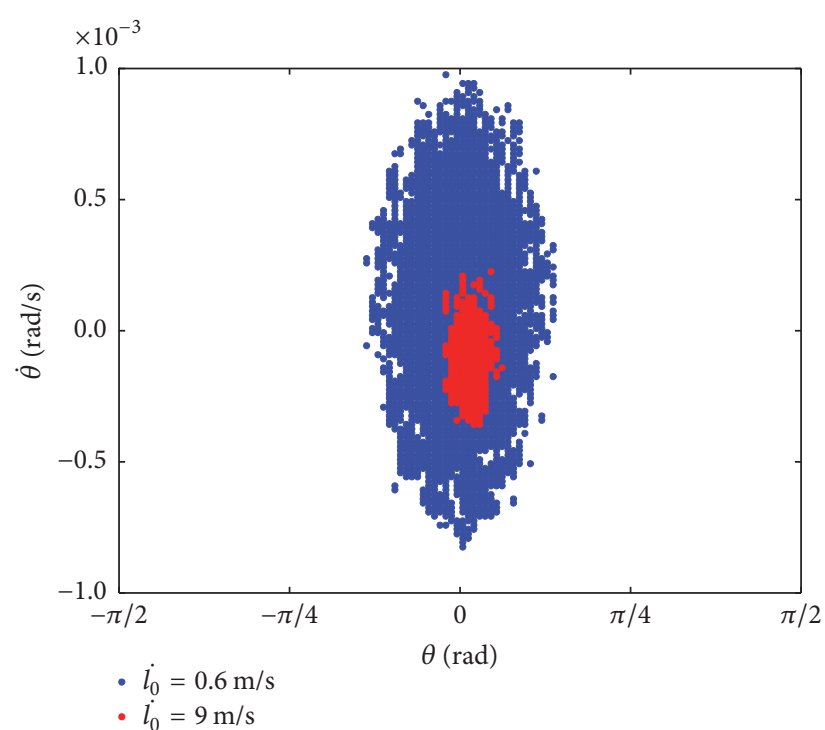

(b) Effect of initial radial velocity of subsatellite on the parameter domain

FIGURE 17: Dependence of parameter domain on radial impact velocities $i<15 \mathrm{~m} / \mathrm{s}$.

acquire the undesired pitch angle and radial impact velocity. In addition, the method of investigating the impact dynamics can be extended to the out-of-plane motions of the tethered system. More complicated nonlinear behaviors appear if the energy dissipation of the space tether caused by impacts is considered.

\section{Conflicts of Interest}

The authors declare that there are no conflicts of interest regarding the publication of this paper.

\section{Acknowledgments}

This study was funded by the National Natural Science Foundation of China (11672125, 11290153), by the Civil Aerospace Pre-Research Project of China (D010305), by the Research Fund of State Key Laboratory of Mechanics and Control of Mechanical Structures (Nanjing University of Aeronautics and Astronautics, MCMS-0116K01), and in part by the Fundamental Research Funds for the Central Universities (NS2016009) and the Priority Academic Program Development of Jiangsu Higher Education Institutions. 


\section{References}

[1] S. Sasaki, K. I. Oyama, N. Kawashima et al., "Results from a series of tethered rocket experiments," Journal of Spacecraft and Rockets, vol. 24, no. 5, pp. 444-453, 1987.

[2] M. Dobrowolny and N. H. Stone, "A technical overview of TSS1: the first tethered-satellite system mission," Il Nuovo Cimento C, vol. 17, no. 1, pp. 1-12, 1994.

[3] R. Hoyt, J. Slostad, and R. Twiggs, "The multi-application survivable tether (MAST) experiment," in Proceedings of the 39th AIAA/ASME/SAE/ASEE Joint Propulsion Conference and Exhibit, Alabama, Ala, USA, July 2003.

[4] B. S. Yu and D. P. Jin, "Deployment and retrieval of tethered satellite system under $J_{2}$ perturbation and heating effect," Acta Astronautica, vol. 67, no. 7-8, pp. 845-853, 2010.

[5] S. Matunaga, O. Mori, and Y. Ohkami, "Dynamics of tethered multibody systems with friction and impact," in Proceedings of the Guidance, Navigation, and Control Conference and Exhibit, Boston, Mass, USA, August 1998.

[6] H. Wen, Z. H. Zhu, D. Jin, and H. Hu, "Constrained tension control of a tethered space-tug system with only length measurement," Acta Astronautica, vol. 119, pp. 110-117, 2016.

[7] H. Wen, Z. H. Zhu, D. Jin, and H. Hu, "Space tether deployment control with explicit tension constraint and saturation function," Journal of Guidance, Control, and Dynamics, vol. 39, no. 4, pp. 915-920, 2016.

[8] A. P. Markeyev, "On the oscillations of a material point suspended from an ideal thread," Journal of Applied Mathematics and Mechanics, vol. 60, no. 2, pp. 240-249, 1996.

[9] C. S. Hsu, "Cell-to-Cell Mapping: a method of global analysis for nonlinear systems," in Applied Mathematical Sciences, SpringerVerlag, 1987.

[10] P. Williams, "Deployment/retrieval optimization for flexible tethered satellite systems," Nonlinear Dynamics, vol. 52, no. 1-2, pp. 159-179, 2008. 


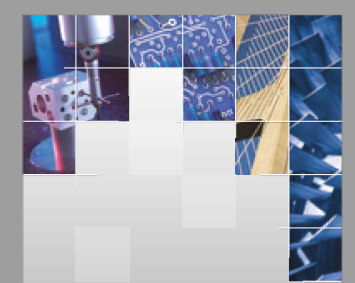

\section{Enfincering}
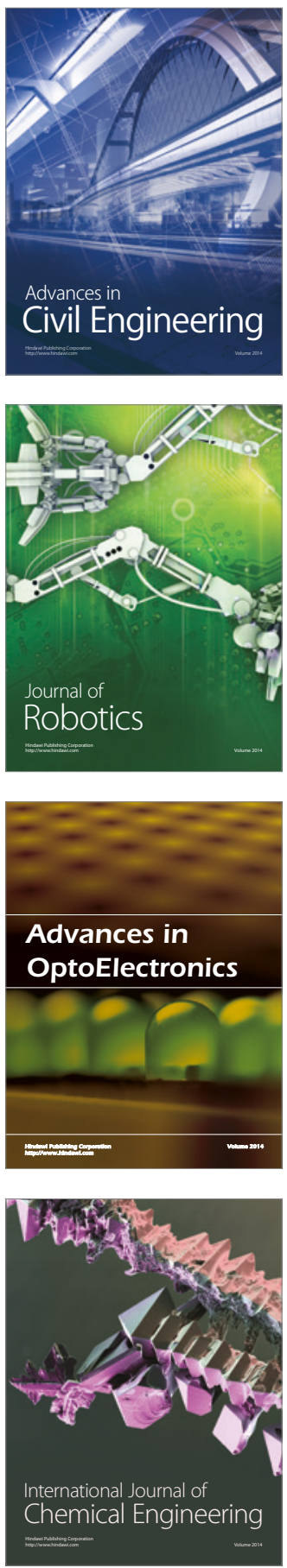

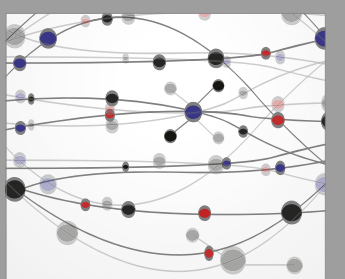

The Scientific World Journal

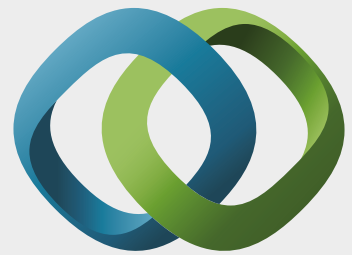

\section{Hindawi}

Submit your manuscripts at

https://www.hindawi.com
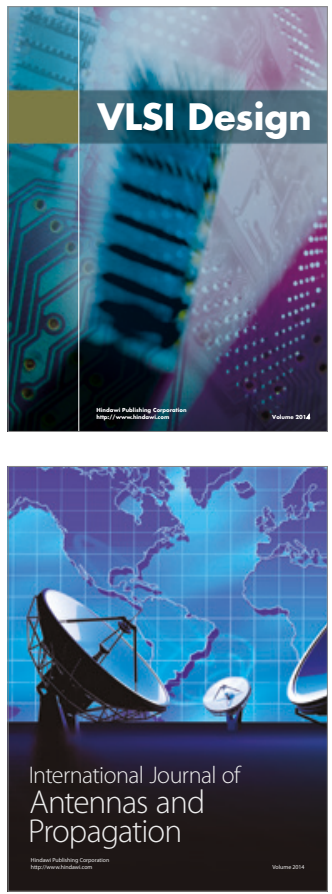

\section{Rotating}

Machinery
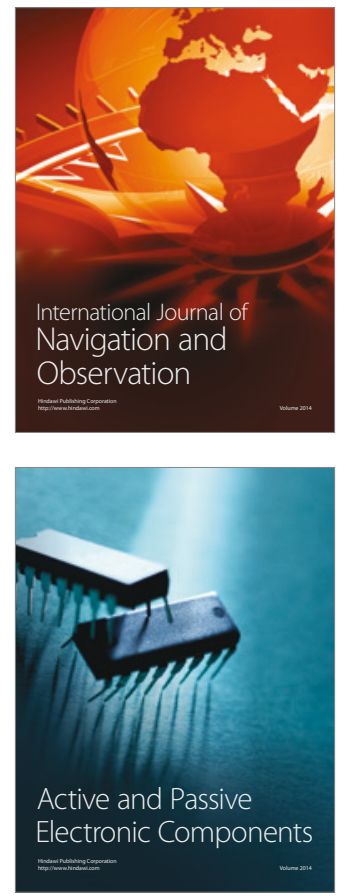
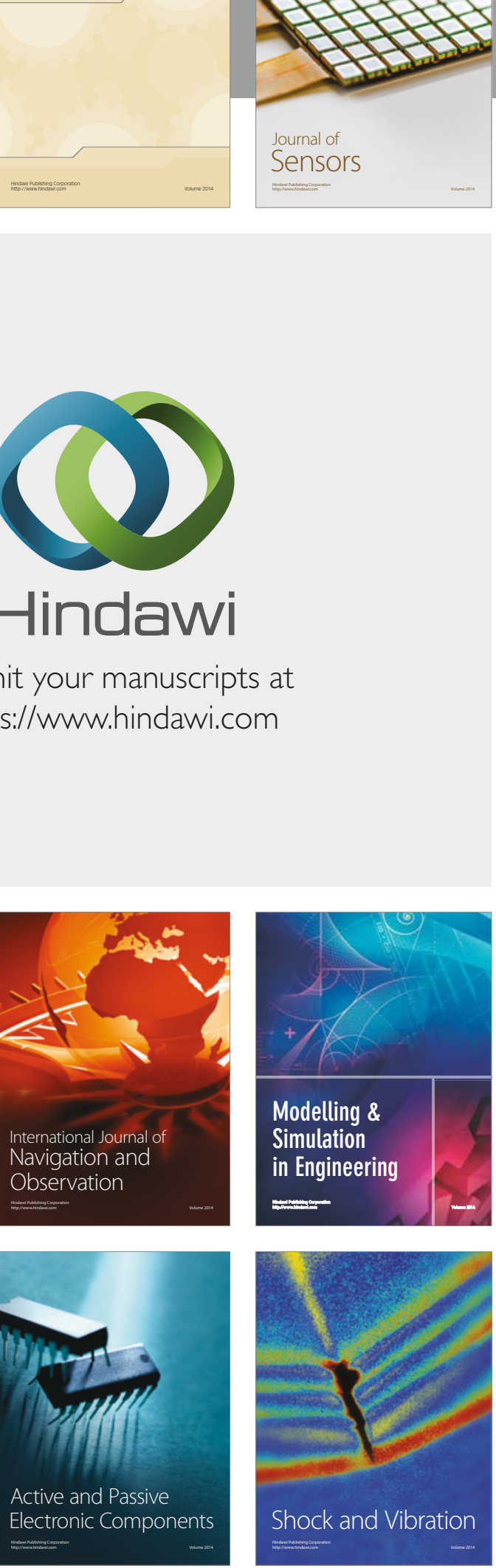
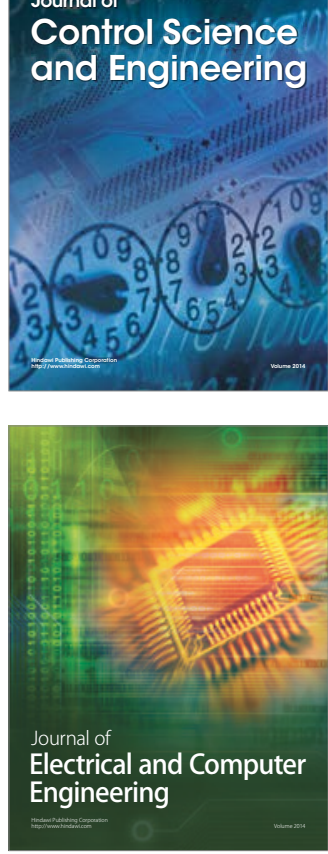

Distributed

Journal of

Control Science

and Engineering
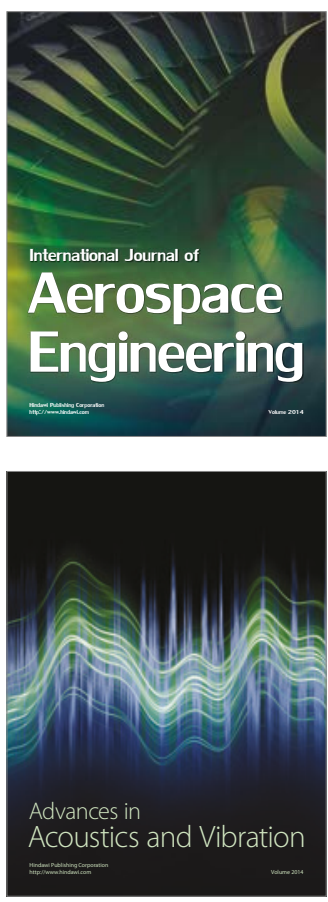

Sensor Networks 